\title{
The Effect of Medetomidine-Ketamine Anesthesia on Hemodynamic Parameters During Hemorrhagic Shock in Minipigs
}

\author{
A. BREZINA ${ }^{1}$, T. DRABEK ${ }^{2}$, H. RIHA ${ }^{1,3}$, J. SCHREIBEROVA $^{4}$, L. HESS $^{5}$ \\ ${ }^{1}$ Department of Anesthesiology and Intensive Care Medicine, Institute for Clinical and \\ Experimental Medicine, Prague, Czech Republic, ${ }^{2}$ Department of Anesthesiology, Safar Center for \\ Resuscitation Research, University of Pittsburgh School of Medicine, Pittsburgh, Pennsylvania, \\ United States of America, ${ }^{3}$ Centre for Cardiovascular Research, Institute of Physiology, Academy \\ of Sciences of the Czech Republic, Prague, Czech Republic, ${ }^{4}$ Department of Anesthesiology and \\ Intensive Care Medicine, University Hospital Hradec Králové, Hradec Králové, Czech Republic, \\ ${ }^{5}$ Centre for Experimental Medicine, Institute for Clinical and Experimental Medicine, Prague, \\ Czech Republic
}

Received November 1, 2009

Accepted March 5, 2010

On-line April 20, 2010

\section{Summary}

Hemorrhagic shock (HS) represents an acute event with high mortality. The optimal combination of anesthetics that would prevent hemodynamic collapse and allow damage control surgery has not yet been determined. We tested the hypothesis that a combination of dissociative anesthetic ketamine with alpha $2_{2}$ agonist medetomidine (MK group, $\mathrm{n}=10$ ) would provide superior hemodynamic control compared to propofol-remifentanil (PR group, $\mathrm{n}=10$ ) during HS in minipigs. A modified Wiggers' model of HS with a target mean arterial pressure (MAP) of $40 \mathrm{~mm} \mathrm{Hg}$ and $2 \mathrm{~h}$ duration was used. All minipigs survived. HS led to a $\sim 50 \%$ decrease in cardiac output in both groups $(P<0.001$ for baseline vs. HS $120 \mathrm{~min}$ ) with no differences between groups. Total volume of removed blood was larger in the MK group $(1321 \pm 133 \mathrm{ml}$ vs. $1111 \pm 246 \mathrm{ml}$ in the PR group, respectively; $P<0.05)$. MAP was higher during the initial phases of HS in the MK group than in PR group ( $P<0.05$ at HS 30-90 min). HR was lower in the MK group at the late phases of HS $(P<0.05$ at HS 60-120 min). In conclusion, medetomidine-ketamine provides a feasible and possibly a more favorable alternative to the propofol-remifentanil combination in our model of HS in minipigs.

\section{Key words}

Hemorrhagic shock • Ketamine • Alpha 2 -agonists • Remifentanil • Propofol

\section{Corresponding author}

Hynek Riha, Department of Anesthesiology and Intensive Care Medicine, Institute for Clinical and Experimental Medicine, Videnska 1958/9, 14021 Prague, Czech Republic. E-mail: hynek.riha@ikem.cz

\section{Introduction}

Hemorrhagic shock (HS) remains a leading cause of preventable death in both civilian and military settings (Alam and Rhee 2007). The acute hypovolemia causes hemodynamic derangement that - if untreated could lead to exsanguination cardiac arrest. While victims of HS require anesthesia to allow for a damage control surgery, virtually all anesthetics adversely affect hemodynamics even in intact patients. The optimal combination of drugs for pre-hospital analgesia and in-hospital anesthesia for HS patients that would preserve hemodynamics has not yet been determined.

Ketamine is a dissociative anesthetic frequently used for sedation, analgesia, or general anesthesia because of its analgetic capacities and favorable hemodynamic effects even in disrupted circulation (Haskins and Patz 1990). Because of its psychomimetic effects, ketamine is often combined with benzodiazepines which, however, in the presence of hypovolemia may 
lead to further decrease of blood pressure (Adams et al. 1985).

Medetomidine, a highly specific alpha 2 -agonist, has a central sympatholytic effect resulting in a decrease in blood pressure and heart rate. In contrast, continuous intravenous infusion of medetomidine increased blood pressure in pigs (Vainio et al. 1992), and also reduced the incidence of psychomimetic effects following ketamine administration (Levanen et al. 1995).

A combination of ketamine and medetomidine in normovolemic animals generally induces bradycardia and severe hypertension (Caulkett et al. 1999, Jalanka et al. 1989, Ko et al. 2000, Sladky et al. 2000, Stegmann and Jago 2006, Tomizawa et al. 1997).

Considering the above-mentioned drug characteristics, we considered the combination of medetomidine-ketamine to be a promising candidate for anesthesia in compromised circulation. In our study we tested the hypothesis that the combination of medetomidine with ketamine has beneficial effects on hemodynamic parameters during HS in minipigs. The control group was anesthetized with commonly used anesthetics propofol and remifentanil.

\section{Methods}

The experimental study was approved by the Institutional Animal Care and Use Committee, Institute for Clinical and Experimental Medicine, Prague, Czech Republic. The experiments were conducted in conformity with the European Convention on Animal Protection. The study was carried out on twenty Minnesota minipigs weighing $35-42 \mathrm{~kg}$ of either sex with randomization to study and control groups ( $\mathrm{n}=10$ per group).

The animals were obtained from a licensed vendor and housed in the facility for at least $72 \mathrm{~h}$ before the experiment. After intramuscular premedication with standard doses of azaperone $(4 \mathrm{mg} / \mathrm{kg})$ and fentanyl $(2 \mu \mathrm{g} / \mathrm{kg})$, an 18-gauge (G) intravenous cannula was placed in the ear vein, and a general anesthesia was induced with intravenous administration of $5 \mathrm{mg} / \mathrm{kg}$ of metomidate. Neuromuscular blockade was not used. The minipigs were then intubated and ventilated with inspired oxygen fraction $\left(\mathrm{FiO}_{2}\right)$ 0.5, tidal volume of $10 \mathrm{ml} / \mathrm{kg}$ with a variable frequency to maintain normocapnia.

Anesthesia before the induction of HS was maintained in both groups by a combination of propofol $(10 \mathrm{mg} / \mathrm{kg} / \mathrm{h})$ and remifentanil $(1 \mu \mathrm{g} / \mathrm{kg} / \mathrm{min})$. Pulmonary artery catheter (model 831, Edwards Lifesciences, CA,
USA; 7.5 French [F]) was advanced via the right internal jugular vein for continuous monitoring of hemodynamic parameters. An $8.5 \mathrm{~F}$ introducer placed in the internal jugular vein was used for blood withdrawal. A $20 \mathrm{G}$ catheter was placed into the femoral artery for invasive blood pressure monitoring.

After instrumentation, the infusion of anesthetics was discontinued for $15 \mathrm{~min}$ to allow for a washout of the anesthetics used during the preparation phase. Animals were then randomized into two groups. In the control group, total intravenous anesthesia (TIVA) was maintained by continuous infusion of propofol and remifentanil (PR group, for the doses see above). In the study group, from the beginning of HS, TIVA was maintained by a combination of medetomidine $(10 \mu \mathrm{g} / \mathrm{kg} / \mathrm{h})$ with ketamine $(5 \mathrm{mg} / \mathrm{kg} / \mathrm{h})$ (MK group).

A modified Wiggers' model of HS was used (Wiggers et al. 1945). In brief, HS was induced by a rapid blood withdrawal to target mean arterial pressure (MAP) of $40 \mathrm{~mm} \mathrm{Hg}$, followed by repeated blood withdrawals every 30 min when necessary to reach MAP $40 \mathrm{~mm} \mathrm{Hg}$ again. The total duration of HS was 2 hours. Surviving animals were then sacrificed with intravenous thiopental combined with a potassium chloride overdose.

Heart rate (HR), MAP, central venous pressure (CVP), mean pulmonary artery pressure (MPAP) and cardiac output $(\mathrm{CO})$ were recorded at baseline and at $30 \mathrm{~min}$ intervals and considered as independent variables. The values were used to calculate stroke volume (SV) and systemic vascular resistance (SVR), which were considered as dependent variables. The total withdrawn blood volume was determined at the end of experiment.

The outcome parameters were statistically evaluated with one-way analysis of variance with Bonferroni's significance level correlation (SPSS v. 16.0, Chicago, IL, USA). $P<0.05$ value was considered statistically significant.

\section{Results}

Baseline (BL) physiologic data were similar in both groups (Table 1). All animals survived in both groups.

HS led to a $\sim 50 \%$ decrease in CO (PR group: BL $3.4 \pm 0.6 \mathrm{l} / \mathrm{min}$, HS $120 \mathrm{~min} 1.7 \pm 0.2 \mathrm{l} / \mathrm{min}$; MK group:

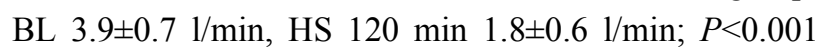
BL vs. HS $120 \mathrm{~min}$ in either group) (Fig. 1). MAP decreased over the time in both groups, but it was better preserved during the initial phases of HS in the MK 
group, reaching statistical significance at HS 30-90 min time points (Fig. 2). HR was lower in the MK group at the late phases of HS (60-120 min) (Fig. 3). SVR was higher in the MK group only at HS $30 \mathrm{~min}$ (MK, $3464 \pm 1049$ dyn.sec/cm ${ }^{5}, P R, 2414 \pm 355$ dyn.sec/ $\mathrm{cm}^{5}$; $P<0.05)$. There were no differences in SV, CVP or MPAP between groups at any time point (data not shown). The total amount of removed blood was larger in the MK group $(1321 \pm 133 \mathrm{ml}$ vs. $1111 \pm 246 \mathrm{ml}$ in the PR group, respectively; $P<0.05$ ). Based on the formula to calculate blood volume of minipigs (Diehl et al. 2001), this represents $53 \%$ of removed blood volume in the MK group vs. $45 \%$ in the PR group, which is consistent with an impending shock state in transition to an irreversible shock state (Wiggers and Ingraham 1946).
Table 1. Baseline hemodynamic parameters of $P R$ and $M K$ minipig groups.

\begin{tabular}{lll}
\hline & PR & MK \\
\hline HR (beats $/ \mathrm{min})$ & $72 \pm 6$ & $71 \pm 18$ \\
MAP (mm Hg) & $92 \pm 8$ & $100 \pm 16$ \\
CO (l/min) & $3.4 \pm 0.6$ & $3.9 \pm 0.7$ \\
CVP (mm Hg) & $7 \pm 2$ & $7 \pm 3$ \\
MPAP (mm Hg) & $24 \pm 3$ & $21 \pm 4$ \\
SVR $\left(\right.$ dyn.sec/ $\left.\mathrm{cm}^{5}\right)$ & $2068 \pm 373$ & $1862 \pm 525$ \\
\hline
\end{tabular}

$\mathrm{PR}$, propofol-remifentanil; $\mathrm{MK}$, medetomidine-ketamine; $\mathrm{HR}$, heart rate; MAP, mean arterial pressure; $\mathrm{CO}$, cardiac output; SVR, systemic vascular resistance; MPAP, mean pulmonary artery pressure; CVP, central venous pressure. Values are mean \pm S.D. No differences between groups.
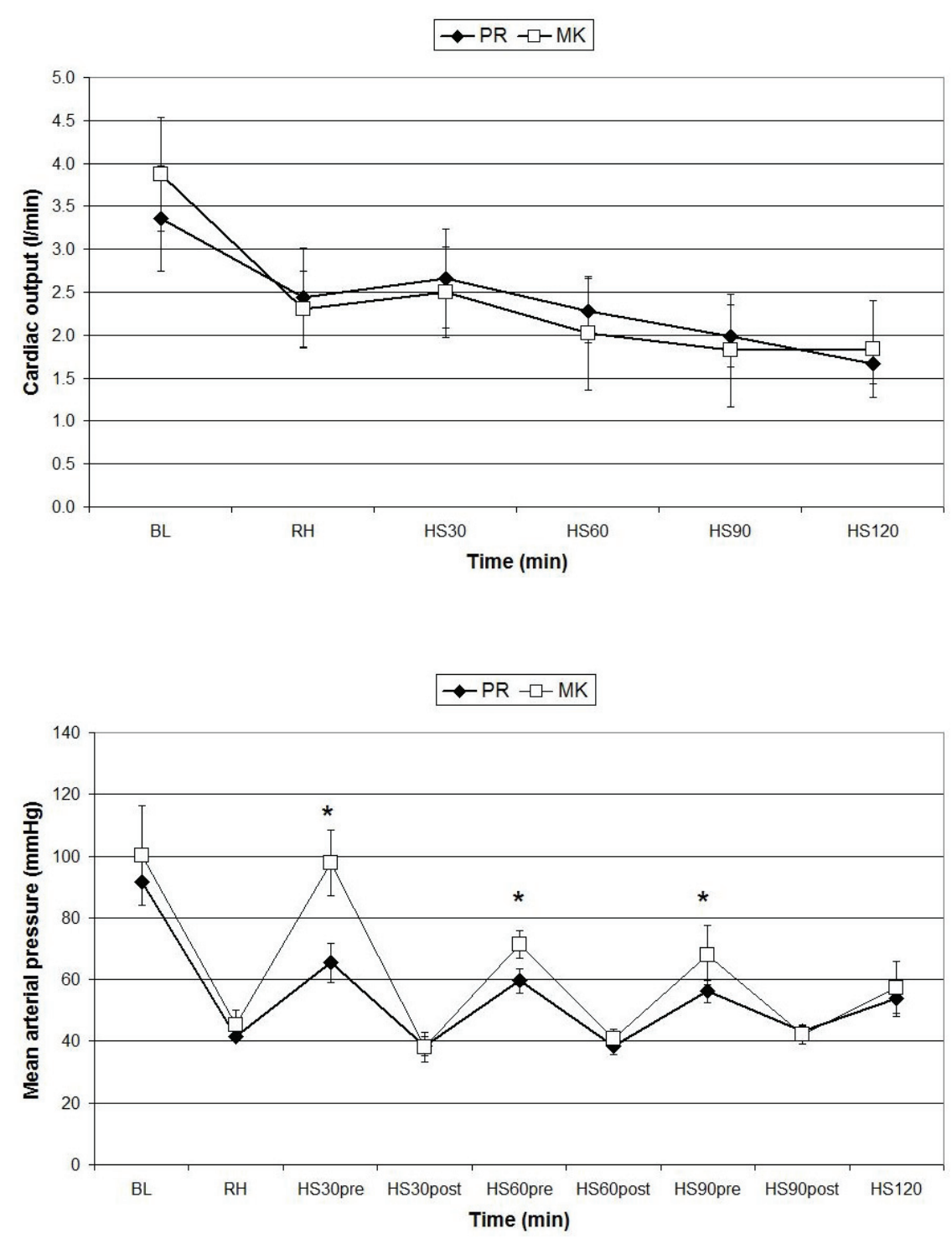

Fig. 1. Cardiac output during hemorrhagic shock. PR, propofolremifentanil; $\mathrm{MK}$, medetomidineketamine; $\mathrm{BL}$, baseline; $\mathrm{RH}$, after initial rapid hemorrhage. $P<0.001 \mathrm{BL}$ vs. HS $120 \mathrm{~min}$ in either group. No differences between groups.

Fig. 2. Mean arterial pressure during hemorrhagic shock. PR, propofolremifentanil; $\mathrm{MK}$, medetomidineketamine; $\mathrm{BL}$, baseline; $\mathrm{RH}$, after initial rapid hemorrhage. $P<0.001 \mathrm{BL}$ vs. HS $120 \mathrm{~min}$ in either group. * $P<0.05$ between groups. 


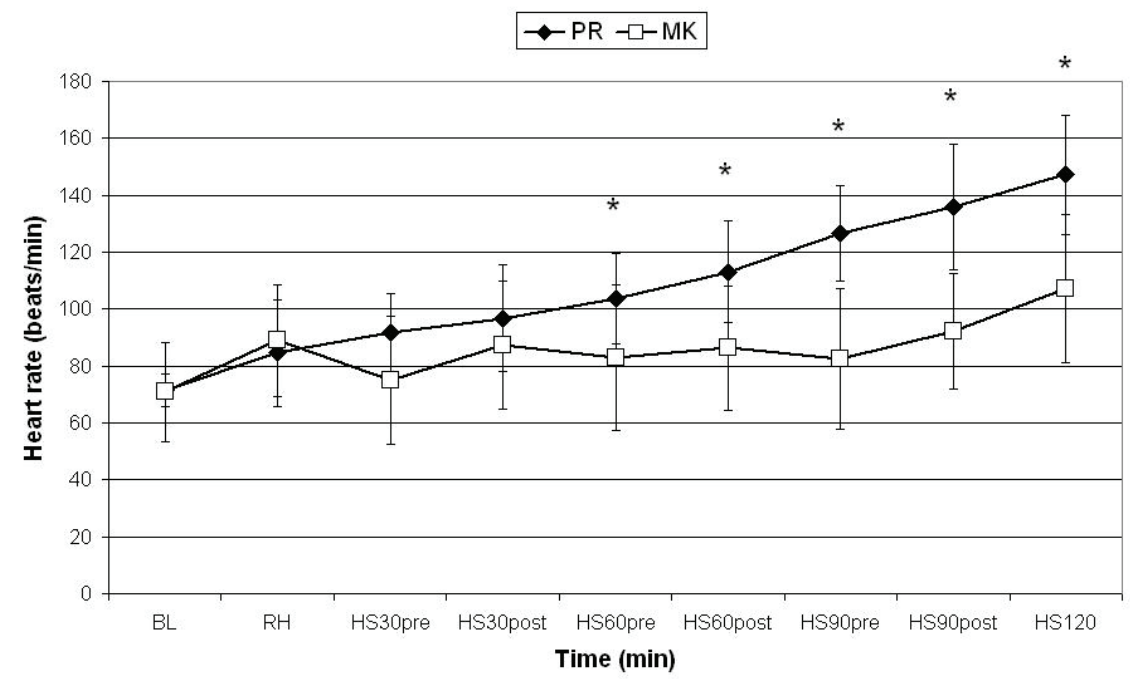

Fig. 3. Heart rate during hemorrhagic shock. PR, propofol-remifentanil; MK, medetomidine-ketamine; $\mathrm{BL}$, baseline; $\mathrm{RH}$, after initial rapid hemorrhage. $P<0.001 \mathrm{BL}$ vs. HS $120 \mathrm{~min}$ in either group. $* P<0.05$ between groups.

\section{Discussion}

We demonstrated that the combination of alpha $2_{2}$ agonist medetomidine with ketamine is characterized by hemodynamic stability during HS in minipigs. The MAP values were significantly higher in the MK group during the whole HS. This was caused by the sympathomimetic effects of ketamine and peripheral vasoconstriction exerted by medetomidine. SVR was constantly higher in the MK group vs. PR group during the entire experiment. HR in the MK group was lower in the late phases of HS (HS 90 and $120 \mathrm{~min}$ ), resulting in an insignificant decrease in $\mathrm{CO}$. This was probably caused by sympatholytic effects of medetomidine. Most importantly, the favorable hemodynamic profile was achieved despite larger volume of removed blood during pressure-targeted HS in the MK group.

A standard treatment for HS victims includes analgesia and sedation. However, currently there is not available a single specific drug designed for analgesia, general anesthesia or sedation during HS. It has been shown that nociceptive stimuli from traumatized area of the body together with pain and fear can augment sympathoadrenergic reactions, thus worsening shock conditions. On the other hand, they can contribute to positive hemodynamic compensatory changes. Unanesthetized dogs subjected to HS showed better survival than their sympathectomized controls (Chien 1964). In contrast, norepinephrine infusion during HS decreased both survival time and rate (Close et al. 1957).

All anesthetics virtually cause ablation of sympathetic activity by decreasing SVR, followed by a decrease in blood pressure. The effects of anesthetics could be even more pronounced in an acutely hypovolemic patient. The choice of an anesthetic could have a significant impact on the outcome of the HS. Peng et al. (2006) showed a $87.5 \%$ survival after 30 or $40 \%$ blood loss in unanesthetized rats vs. $0 \%$ survival in those anesthetized with sodium pentobarbital. Longnecker and Sturgill (1976) documented superior acute and long-term survival rates in a rat HS model with ketamine compared to halothane, fluroxene or pentobarbital. This effect was accompanied by a relative absence of pathologic changes in the small intestine and liver, suggesting preferential shifting of perfusion to the gut.

Ketamine, an N-methyl-D-aspartate (NMDA)antagonist, has unique characteristics in comparison with commonly used anesthetics. It increases sympathoadrenal reactions, SVR and venous return. Spontaneous breathing and protective reflexes of upper airways are partially preserved. Ketamine has been successfully used since the 1970s in hypovolemic patients or in patients with HS (Bond and Davies 1974, Chasapakis et al. 1973). In these reports, a combination of ketamine with pancuronium, a neuromuscular blocking agent with vagolytic properties, has been used. However, further studies showed that the use of pancuronium in decompensated HS is deleterious (Christian et al. 1979). A favorable effect on survival from HS in rats was reported using a combination of ketamine and alpha 2 -agonist clonidine (Kaukinen 1978).

Psychomimetic effects associated with ketamine are often unpleasant. The combination of ketamine with benzodiazepines to ameliorate this phenomenon is effective but not devoid of side-effects, namely further reduction in SVR and ensuing hypotension in hypovolemic patients (Adams et al. 1985).

Alpha $_{2}$-agonists are used for their antihypertensive properties. They elicit a dual response: the 
first phase is characterized by a transient hypertension (caused by a peripheral vasoconstriction) followed by hypotension in the second phase (caused by a central vasodilation). The overall blood pressure response to alpha $_{2}$-agonists depend on several factors: dose, route of administration, selectivity for alpha $2_{2}$-receptors, and concurrently given drugs (Sinclair 2003). With intravenous application of ketamine, continuous infusion of alpha ${ }_{2}$-agonist antagonizes sympathomimetic effects of ketamine, and the net cardiovascular effects depend on the balance between the effects of both drugs.

Medetomidine is a highly specific alpha ${ }_{2}$-agonist with short half-life of 2.5 hours (Scheinin and Schwinn 1992). In continuous infusion it was also shown to attenuate the psychomimetic effects of ketamine, although to a lesser degree than midazolam (Levanen et al. 1995). Vainio et al. (1992) published detailed hemodynamic data in minipigs using combination of medetomidine-ketamine anesthesia. MAP increased from $116 \mathrm{~mm} \mathrm{Hg}$ to $142 \mathrm{~mm} \mathrm{Hg}$ and then slowly decreased to baseline values during $60 \mathrm{~min}$. SVR increased three-fold. CO decreased from $1.8 \pm 0.7 \mathrm{l} / \mathrm{min}$ to $0.4 \pm 0.3 \mathrm{l} / \mathrm{min}$. The plasma levels of norepinephrine and epinephrine significantly decreased. In humans, clonidine significantly reduced hypertension caused by ketamine during inhalational anesthesia (Tanaka and Nishikawa 1994) or at the time of endotracheal intubation (Munro et al. 1993). Dexmedetomidine and ketamine combination has been successfully used in pediatric patients undergoing cardiac catheterization with little hemodynamic variations (Barton et al. 2008, Mester et al. 2008).

Medetomidine is a racemate designed for veterinary medicine. Dexmedetomidine is eight-times more selective for alpha $_{2}$-receptors compared to a partial alpha ${ }_{2}$-agonist clonidine (Scheinin et al. 1989). The difference in selectivity for alpha ${ }_{2}$-receptors between medetomidine and clinically used dexmedetomidine has not been studied.

Thus, we considered the use of medetomidine in minipigs for our study as a logical and a reasonable choice that would adequately mirror a clinical scenario. Nevertheless, the differences in hemodynamic response to alpha $a_{2}$-agonists among species should be taken into consideration while interpreting the results of our study.

The importance of maintaining pre-set levels of MAP vs. CO in HS patients has been a matter of ongoing debate and large controversy. In our study, CO decreased by $\sim 50 \%$ in both groups, while MAP was better preserved in the MK group, suggesting greater hemodynamic stability in the MK group. In addition, the combination of medetomidine with ketamine - despite blunting the physiologic response of increase in HR due to hypovolemia - maintained $\mathrm{CO}$ with lower HR, i.e. by higher stroke volume due to better left ventricular filling despite higher blood loss compared with the combination of propofol-remifentanil.

Our study has several limitations. There were no deaths in either group suggesting that despite no fluid resuscitation, the severity of the insult was only moderate. We did not monitor biochemical markers of organ injury that would provide an additional information of the metabolic derangements of the animals. Selected metabolic parameters, e.g. lactate, base deficit or $\mathrm{pH}$, were shown to have better predictive value than classic hemodynamic parameters used traditionally as outcome parameters in HS studies (Rixen et al. 2001).

Our experiment was terminated at the end of HS. We did not assess the effect of the anesthetic combinations on the long-term survival following resuscitation. It has been shown recently that a combination of an alpha $a_{2}$-agonist dexmedetomidine with ketamine mitigates acute lung injury in rats subjected to HS (Yang et al. 2009). A protective dose-dependent effects of propofol that mitigated end-organ injury after HS have been described across species (Lee et al. 2008, Lee et al. 2009, Yao et al. 2009). Similar effect was found with fentanyl (Yao et al. 2009). Thus, all drugs used in our study could play a distinct role in ameliorating ischemia-reperfusion injury during the resuscitation phase and ultimately affect long-term outcome from HS.

We also assumed that the doses of drugs and their respective combinations were equipotent but this was not verified in an independent study. The pharmacologic protocols allowed to perform the study in nonparalyzed animals, confirming an adequate anesthesia depth in both groups. We did not adjust the doses of individual drugs during the HS. HS was shown to alter the pharmacokinetics of remifentanil, suggesting that less remifentanil would be required to maintain a target plasma concentration (Johnson et al. 2001). Both pharmacokinetics and pharmacodynamics of propofol were changed in the setting of HS (Johnson et al. 2003, Kurita et al. 2009). It should be noted that all abovementioned pharmacological studies used similar Wiggers“ model of HS. In a separate study, hypnotic potency of propofol was shown to be increased even in an 
isovolemic model of HS in humans with preserved $\mathrm{CO}$ (Takizawa et al. 2006).

We chose not to include a group that would be treated with a volatile anesthetic. Instead, we focused on a combination of intravenous agents that could be used in the prehospital settings or in locations with limited resources. Other combinations of drugs, e.g. ketaminemidazolam, represent another alternative. However, the deleterious effects of midazolam in compromised circulation (Adams et al. 1985) lessen the enthusiasm for its use in this setting. Further studies using novel drugs with enhanced profile, e.g. dexmedetomidine or S-ketamine, devoid of psychomimetic properties, are thus warranted.

In conclusion, a combination of medetomidine and ketamine during hemorrhagic shock in minipigs resulted in a higher blood pressure and decreased heart rate compared to propofol-remifentanil combination.
There were no differences in cardiac output between the groups. Hemodynamic stability was maintained despite larger volume of removed blood in the medetomidineketamine group. Our results suggest that medetomidineketamine provide a feasible and possibly a more favorable alternative to the propofol-remifentanil combination in our model of hemorrhagic shock in minipigs.

\section{Conflict of Interest}

There is no conflict of interest.

\section{Acknowledgements}

This study was supported by the Ministry of Health of the Czech Republic within the research program MZO 00023001 "Research on cardiovascular diseases, diabetes mellitus and transplantation of vital organs".

\section{References}

ADAMS P, GELMAN S, REVES JG, GREENBLATT DJ, ALVIS JM, BRADLEY E: Midazolam pharmacodynamics and pharmacokinetics during acute hypovolemia. Anesthesiology 63: 140-146, 1985.

ALAM HB, RHEE P: New developments in fluid resuscitation. Surg Clin North Am 87: 55-72, 2007.

BARTON KP, MUNOZ R, MORELL VO, CHRYSOSTOMOU C: Dexmedetomidine as the primary sedative during invasive procedures in infants and toddlers with congenital heart disease. Pediatr Crit Care Med 9: 612-615, 2008.

BOND AC, DAVIES CK: Ketamine and pancuronium for the shocked patient. Anaesthesia 29: 59-62, 1974.

CAULKETT NA, CATTET MR, CAULKETT JM, POLISCHUK SC: Comparative physiologic effects of telazol, medetomidine-ketamine, and medetomidine-telazol in captive polar bears (Ursus maritimus). J Zoo Wildl Med 30: 504-509, 1999.

CHASAPAKIS G, KEKIS N, SAKKALIS C, KOLIOS D: Use of ketamine and pancuronium for anesthesia for patients in hemorrhagic shock. Anesth Analg 52: 282-287, 1973.

CHIEN S: Role of sympathetic nervous system in surviving acute hemorrhage. Am J Physiol 206: 21-24, 1964.

CHRISTIAN CM, NARAGHI M, ADRIANI J: Adverse effects of pancuronium in patients with hemorrhagic shock. South Med J 72: 1113-1115, 1979.

CLOSE AS, WAGNER JA, KLOEHN RA Jr, KORY RC: The effect of norepinephrine on survival in experimental acute hemorrhagic hypotension. Surg Forum 8: 22-26, 1957.

DIEHL KH, HULL R, MORTON D, PFISTER R, RABEMAMPIANINA Y, SMITH D, VIDAL JM, VAN DE VORSTENBOSCH C: A good practice guide to the administration of substances and removal of blood, including routes and volumes. J Appl Toxicol 21: 15-23, 2001.

HASKINS SC, PATZ JD: Ketamine in hypovolemic dogs. Crit Care Med 18: 625-629, 1990.

JALANKA H, SKUTNABB K, DAMSTEN Y: Preliminary results on the use of medetomidine-ketamine combinations in the dog. Acta Vet Scand Suppl 85: 125-127, 1989.

JOHNSON KB, KERN SE, HAMBER EA, MCJAMES SW, KOHNSTAMM KM, EGAN TD: Influence of hemorrhagic shock on remifentanil: a pharmacokinetic and pharmacodynamic analysis. Anesthesiology 94: 322-332, 2001. 
JOHNSON KB, EGAN TD, KERN SE, WHITE JL, MCJAMES SW, SYROID N, WHIDDON D, CHURCH T: The influence of hemorrhagic shock on propofol: a pharmacokinetic and pharmacodynamic analysis. Anesthesiology 99: 409-420, 2003.

KAUKINEN S: Effects of antihypertensive medication on the cardiovascular response to ketamine in rats. Acta Anaesthesiol Scand 22: 437-446, 1978.

KO JC, FOX SM, MANDSAGER RE: Sedative and cardiorespiratory effects of medetomidine, medetomidinebutorphanol, and medetomidine-ketamine in dogs. J Am Vet Med Assoc 216: 1578-1583, 2000.

KURITA T, TAKATA K, MORITA K, MORISHIMA Y, URAOKA M, KATOH T, SATO S: The influence of hemorrhagic shock on the electroencephalographic and immobilizing effects of propofol in a swine model. Anesth Analg 109: 398-404, 2009.

LEE CJ, SUBEQ YM, LEE RP, WU WT, HSU BG: Low-dose propofol ameliorates haemorrhagic shock-induced organ damage in conscious rats. Clin Exp Pharmacol Physiol 35: 766-774, 2008.

LEE CJ, LEE RP, SUBEQ YM, LEE CC, PENG TC, HSU BG: Propofol protects against hemorrhagic shock-induced organ damage in conscious spontaneously hypertensive rats. Biol Res Nurs 11: 152-162, 2009.

LEVANEN J, MAKELA ML, SCHEININ H: Dexmedetomidine premedication attenuates ketamine-induced cardiostimulatory effects and postanesthetic delirium. Anesthesiology 82: 1117-1125, 1995.

LONGNECKER DE, STURGILL BC: Influence of anesthetic agent on survival following hemorrhage. Anesthesiology 45: 516-521, 1976.

MESTER R, EASLEY RB, BRADY KM, CHILSON K, TOBIAS JD: Monitored anesthesia care with a combination of ketamine and dexmedetomidine during cardiac catheterization. Am J Ther 15: 24-30, 2008.

MUNRO HM, SLEIGH JW, PAXTON LD: The cardiovascular response to ketamine: the effects of clonidine and lignocaine. Acta Anaesthesiol Scand 37: 75-78, 1993.

PENG TC, LIAO KW, LAI HL, CHAO YF, CHANG FM, HARN HJ, LEE RP: The physiological changes of cumulative hemorrhagic shock in conscious rats. J Biomed Sci 13: 385-394, 2006.

RIXEN D, RAUM M, HOLZGRAEFE B, SAUERLAND S, NAGELSCHMIDT M, NEUGEBAUER EA: A pig hemorrhagic shock model: oxygen debt and metabolic acidemia as indicators of severity. Shock 16: 239-244, 2001.

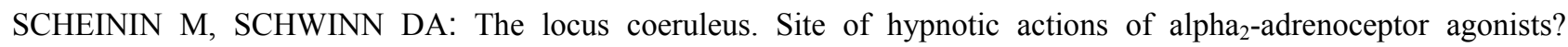
Anesthesiology 76: 873-875, 1992.

SCHEININ H, VIRTANEN R, MACDONALD E, LAMMINTAUSTA R, SCHEININ M: Medetomidine - a novel alpha $_{2}$-adrenoceptor agonist: a review of its pharmacodynamic effects. Prog Neuropsychopharmacol Biol Psychiatry 13: 635-651, 1989.

SINCLAIR MD: A review of the physiological effects of alpha ${ }_{2}$-agonists related to the clinical use of medetomidine in small animal practice. Can Vet J 44: 885-897, 2003.

SLADKY KK, KELLY BT, LOOMIS MR, STOSKOPF MK, HORNE WA: Cardiorespiratory effects of four alpha2adrenoceptor agonist-ketamine combinations in captive red wolves. J Am Vet Med Assoc 217: 1366-1371, 2000.

STEGMANN GF, JAGO M: Cardiopulmonary effects of medetomidine or midazolam in combination with ketamine or tiletamine/zolazepam for the immobilisation of captive cheetahs (Acinonyx jubatus). J S Afr Vet Assoc 77: 205-209, 2006.

TAKIZAWA E, TAKIZAWA D, HIRAOKA H, SAITO S, GOTO F: Disposition and pharmacodynamics of propofol during isovolaemic haemorrhage followed by crystalloid resuscitation in humans. Br J Clin Pharmacol 61: 256-261, 2006.

TANAKA M, NISHIKAWA T: Oral clonidine premedication attenuates the hypertensive response to ketamine. $B r J$ Anaesth 73: 758-762, 1994.

TOMIZAWA N, TOMITA I, NAKAMURA K, HARA S: A comparative study of medetomidine-butorphanol-ketamine and medetomidine-ketamine anaesthesia in dogs. Zbl Veterinarmed A 44: 189-194, 1997.

VAINIO OM, BLOOR BC, KIM C: Cardiovascular effects of a ketamine-medetomidine combination that produces deep sedation in Yucatan mini swine. Lab Anim Sci 42: 582-588, 1992. 
WIGGERS HC, INGRAHAM RC: Hemorrhagic shock: definition and criteria for its diagnosis. J Clin Invest 25: 30-36, 1946.

WIGGERS HC, INGRAHAM RC, DILLE J: Hemorrhagic-hypotension shock in locally anesthetized dogs. Am $J$ Physiol 143: 126-133, 1945.

YANG CH, TSAI PS, WANG TY, HUANG CJ: Dexmedetomidine-ketamine combination mitigates acute lung injury in haemorrhagic shock rats. Resuscitation 80: 1204-1210, 2009.

YAO L, KUAI J, LIU X, YU D, YANG Y, TAO L, ZENG X, CHAI W: Pretreatment with fentanyl and propofol attenuates myocardial injury in rabbits with acute hemorrhagic shock. $J$ Surg Res 155: 25-31, 2009. 